\title{
SOME RECENT DEVELOPMENTS IN THE CALCULUS OF VARIATIONS.*
}

BY PROFESSOR GILBERT AMES BLISS.

IT is my purpose to speak this afternoon of a part of the theory of the calculus of variations which has aroused the interest and taxed the ingenuity of a sequence of mathematicians beginning with Legendre, and extending by way of Jacobi, Clebsch, Weierstrass, and a numerous array of others, to the present time. The literature of the subject is very large and is still growing. I was discussing recently the title of this address with a fellow mathematician who remarked that he was not aware that there had been any recent progress in the calculus of variations. This was a very natural suspicion, I think, in view of the fact that the attention of most mathematicians of the present time seems irresistibly attracted to such subjects as integral equations and their generalizations, the theory of definite integration, and the theory of functions of lines. It is indeed in these latter domains that the activities especially characteristic of the present era are centered, and the progress already made in them, and the further progress inevitable in the near future, will doubtless be sufficient alone to insure for our generation of mathematical workers a noteworthy place in the history of the science.

While speaking of present day mathematical tendencies I should like to take occasion to mention a remark which has been made to me a number of times by persons who are interested in mathematics primarily for its applications. The feeling of some of these scientists seems to be that mathematical research in America is drawing farther and farther away from the forms of mathematics most immediately useful in related subjects, and they wish to attract the attention and stimulate the interest of mathematicians in the directions of these intermediate domains. We should indeed sympathize heartily with this desire. It is not at all to be regarded

* Address of the retiring chairman of the Chicago Section of the American Mathematical Society, read at the joint meeting of the Chicago and Southwestern Sections, the Missouri Section of the Mathematical Association of America, and Section A of the American Association for the Advancement of Science, at St. Louis, December 30, 1919. 
as unfortunate that so much research is being carried on in this country in the purely mathematical field, for one must not forget that the history of mathematics presents repeated instances of pure mathematics of former times which has become applied mathematics of to-day. Furthermore from the educational standpoint it is most important to note that the spirit of research itself, apart from the results attained, is the guarantee in any individual of continuous self-instruction and development, and consequent increase in value to his community. It does seem unfortunate, however, that for some reason the number of people in this country interested in the adaptation of mathematical theories to the pressing contemporary problems of the applications has been relatively small. I am hoping sincerely that cooperation of mathematicians with scientists in other fields will presently be effective in remedying this defect. The mathematical community should assist in every possible way in developing strong applied mathematical research centers especially where beginnings have already been made, in encouraging graduate students to investigations in the fields intermediate between mathematics and the neighboring sciences, and in the discovery and dissemination among maturer workers of information concerning the applied mathematical problems now awaiting solution. In this connection let me also suggest that mathematicians would do well to inquire searchingly into the advantages or disadvantages of cooperative work among themselves, as well as with scientists in other fields. Cooperation seems to be the watchword of the time, and we should not be the last to recognize it. I am myself not yet convinced of the economy of group effort as over against individual effort in mathematical research, but I believe it important that in the near future we should have some conclusive information about it.

Let me return, however, to the original topic of my discourse which was to have been a part of the theory of the calculus of variations. I should like very much to dwell on some of the modifications which have been made possible by recent progress in the domains of real function theory mentioned in my first paragraph above, but it would be impossible in the limited time at my disposal. Instead I wish to speak of a new method of treating the second variation in the calculus of variations, a method which has rested partly developed 
in my mind for some time, and which has recently turned out to be effective in simplifying the theory of the second variation and coordinating the complicated and extensive literature of the subject.

The simplest problem of the calculus of variations, the one to which my expository remarks this afternoon must be for the most part restricted, is that of determining a curve

$$
y=y(x) \quad\left(x_{1} \leqq x \leqq x_{2}\right)
$$

in the $x y$-plane, joining two fixed points $\left(x_{1}, y_{1}\right)$ and $\left(x_{2}, y_{2}\right)$, and minimizing an integral

$$
J(C)=\int_{x_{1}}^{x_{2}} f\left(x, y(x), y^{\prime}(x)\right) d x .
$$

In order to obtain conditions which must be satisfied by a minimizing arc let us consider the values of the integral along the curves of a family of the form

$$
\bar{y}=y(x)+\alpha \eta(x) \quad\left(x_{1} \leqq x \leqq x_{2}\right),
$$

where $\alpha$ is a constant to be varied at pleasure and $\eta(x)$ is a function which vanishes at $x_{1}$ and $x_{2}$, so that $\eta\left(x_{1}\right)=\eta\left(x_{2}\right)=0$. All of the curves of this family pass through the end points of the original curve $C$, on account of the last condition imposed upon the $\eta(x)$, and it is clear that the function of $\alpha$,

$$
J(\alpha)=\int_{x_{1}}^{x_{2}} f\left(x, y+\alpha \eta, y^{\prime}+\alpha \eta^{\prime}\right) d x,
$$

must have a minimum for $\alpha=0$, so that by the usual theory of maxima and minima the conditions

$$
\begin{aligned}
J^{\prime}(0) & =\int_{x_{1}}^{x_{2}}\left(f_{y} \eta+f_{y^{\prime}} \eta^{\prime}\right) d x=0, \\
J^{\prime \prime}(0) & =\int_{x_{1}}^{x_{2}}\left(f_{y y} \eta^{2}+2 f_{y y^{\prime}} \eta \eta^{\prime}+f_{y^{\prime} y^{\prime} \eta^{\prime 2}}\right) d x \\
& =\int_{x_{1}}^{x_{2}} 2 \Omega\left(x, \eta, \eta^{\prime}\right) d x \geqq 0
\end{aligned}
$$

must be satisfied for every choice of the function $\eta(x)$ vanishing at $x_{1}$ and $x_{2}$. Here the arguments of the derivatives of $f$ are the $y(x)$ and $y^{\prime}(x)$ which belong to the curve $C$, and the symbol 
$2 \Omega\left(x, \eta, \eta^{\prime}\right)$ is merely a notation for the quadratic form which appears in the integrand of $J^{\prime \prime}(0)$. The expressions (2) and (3) are called, respectively, the first and second variations of the integral $J$ along the arc $C$.

The first term of the first variation can be integrated by parts in simple fashion giving the equation

$$
J^{\prime}(0)=\int_{x_{1}}^{x_{2}}\left(f_{y^{\prime}}-\int_{x_{1}}^{x} f_{y} d x\right) \eta^{\prime} d x=0
$$

which must hold for every $\eta(x)$ vanishing at $x_{1}$ and $x_{2}$, and it is provable from this condition that there must be a constant $c$ such that along the minimizing arc $C$

$$
f_{y^{\prime}}=\int_{x_{1}}^{x} f_{y} d x+c .
$$

This is a very important equation from which can be deduced several consequences. In the first place the derivative $f_{y^{\prime}}$ must be continuous all along the minimizing arc $C$, since the second member of the equation has this property, so that at each corner point $\left(x_{0}, y_{0}\right)$ of $C$ the equation

$$
f_{y^{\prime}}\left(x_{0}, y_{0}, y^{\prime}\left(x_{0}-0\right)\right)=f_{y^{\prime}}\left(x_{0}, y_{0}, y^{\prime}\left(x_{0}+0\right)\right)
$$

must be satisfied by the two slopes $y^{\prime}\left(x_{0}-0\right), y^{\prime}\left(x_{0}+0\right)$ of the arc $C$ toward the right and left from the corner. In the second place it is provable at once by differentiating the two sides of the equation that the differential equation

$$
\frac{d}{d x} f_{y^{\prime}}=f_{y}
$$

must be satisfied everywhere on $C$. Finally it follows from the equation (4) by somewhat more complicated reasoning that the minimizing arc, supposed at first to have only a first derivative $y^{\prime}$ elsewhere than at corners, must have also higher derivatives, at least at every point at which the condition $f_{y^{\prime} y^{\prime}} \neq 0$ is satisfied, i.e. at every point at which the differential equation (6) is non-singular.

The differential equation (6) of the minimizing arc is now usually ascribed to Euler,* though for a long time it was called

* Methodus inveniendi, etc. (1744), Chapter II, Section 21; translated into German in Ostwald's Klassiker der exakten Wissenschaften, No. 46, p. 53. 
the Lagrange equation, because Lagrange adopted it and used it so widely. The integration by parts which I have mentioned in treating the first variation is due to du Bois Reymond.* The corner condition (5) was deduced by Weierstrass for the parametric case in his lectures as early as 1865 , but was rediscovered by Erdmann in $1877 \dagger$ before the results of Weierstrass were published. The differentiability properties of the minimizing arc were studied by Hilbertł by means of the equation (4), but I think that the derivation of them from this equation by a very simple application of implicit function theorems was due to Mason and myself. $\$$

Before proceeding to the theory of the second variation which is my ultimate goal, I must mention two further contributions to the theory by Weierstrass, familiar to those of you who have studied the calculus of variations, but perhaps not so to others. In his lectures of 1879 Weierstrass introduced his so-called $E$-function

$E\left(x, y, y^{\prime}, \bar{y}^{\prime}\right)=f\left(x, y, \bar{y}^{\prime}\right)-f\left(x, y, y^{\prime}\right)-\left(\bar{y}^{\prime}-y^{\prime}\right) f_{y^{\prime}}\left(x, y, y^{\prime}\right)$.

It is clearly the difference of $f\left(x, y, \bar{y}^{\prime}\right)$ and the sum of the first two terms obtained by expanding this function by Taylor's formula in powers of $\bar{y}^{\prime}-y^{\prime}$. With the help of very simple types of comparison curves he established the further condition that

$$
E\left(x, y, y^{\prime}, \bar{y}^{\prime}\right) \geqq 0
$$

at every set of elements $\left(x, y, y^{\prime}, \bar{y}^{\prime}\right)$ for which $x, y, y^{\prime}$ belongs to the arc $C$ and $\bar{y}^{\prime}$ is arbitrary. This was a new and important result, for all the necessary conditions deduced before his time had involved only comparison curves which hugged closely the original arc $C$ both in position and direction, and he was the first to show that the admission of variations which departed widely in direction, though not in position, from the minimizing arc $C$ gives rise to this further restrictive property.

But he added to the theory in other important respects also, only one of which I can here lay before you. The equation (6), after the differentiation with respect to $x$ has

* Math. Annalen, vol. 15 (1879), p. 313.

$\dagger$ Journal für Mathematik, vol. 82 (1877), p. 21.

$\ddagger$ Göttinger Nachrichten, 1900, p. 253.

$\$$ "The properties of curves in space which minimize a definite integral,"

Trans. Amer. Math. Society, vol. 9 (1908), p. 440. 
been performed, has the form

$$
f_{y^{\prime} x}+f_{y^{\prime} y} y^{\prime}+f_{y^{\prime} y^{\prime}} y^{\prime \prime}=f_{y},
$$

which shows it to be of the second order, and its solutions form a two-parameter family of curves which are called the extremals of the problem. Weierstrass considered especially one-parameter families of such extremals of the form

$$
y=\varphi(x, a),
$$

containing the particular arc $C$, whose minimizing properties are under investigation, for a particular parameter value $a_{0}$. If a family of this sort can be found which simply covers a neighborhood $F$ of $C$, then the region $F$ with its covering of extremals is called a field about $C$. By saying that $F$ is simply covered by the family (7) it is meant that through each point of the region there passes one and but one extremal of the family, so that with each point $(x, y)$ of the field $F$ there is associated a unique extremal slope $p(x, y)$. If now $\bar{C}$ is an arbitrary curve

$$
y=\bar{y}(x) \quad\left(x_{1} \leqq x \leqq x_{2}\right)
$$

in the field $F$ joining the end points of $C$ then Weierstrass showed that the difference $J(\bar{C})-J(C)$ of the values of $J$ along the two arcs is expressible by the formula

$$
J(\bar{C})-J(C)=\int_{x_{1}}^{x_{2}} E\left(x, \bar{y}, p(x, \bar{y}), \bar{y}^{\prime}\right) d x
$$

in which $\bar{y}$ stands always for the function $\bar{y}(x)$ defining the arc $\bar{C}$.

It is clear from this formula that $J(C)$ will surely be a minimum if the arc $C$ can be imbedded in a field $F$ of the type described above, and if the integrand in the second member of the formula (8) is always positive along arcs of the type of $\bar{C}$ in the field. I should be giving a resume of most of the theory of the calculus of variations if I should attempt to describe in detail the conditions under which the field $F$ can be constructed, and which insure the minimizing properties of $C$. They are, however, well known, and for this simple case are not difficult to develop and present. 
Hilbert* devised a very elegant method of establishing the formula (8) which has now superseded with most writers the original "Weierstrass construction" by means of which Weierstrass himself established his result. Hilbert showed that the value of the integral

$$
J^{*}(\bar{C})=\int_{x_{1}}^{x_{2}}\left\{f\left(x, \bar{y}, \bar{y}^{\prime}\right)+\left[\bar{y}^{\prime}-p(x, \bar{y})\right] f_{y^{\prime}}(x, \bar{y}, p(x, \bar{y}))\right\} d x
$$

is independent of the path $\bar{C}$ chosen in the field joining two fixed points, and that along an extremal arc of the field $J^{*}(C)=J(C)$, since along such an arc the differential equation $y^{\prime}=p(x, y)$ is always satisfied. It follows then at once that

$$
\begin{aligned}
J(\bar{C})-J(C)=J(\bar{C})-J^{*}(C)= & J(\bar{C})-J^{*}(\bar{C}) \\
& =\int_{x_{1}}^{x_{2}} E\left(x, \bar{y}, p(x, \bar{y}), \bar{y}^{\prime}\right) d x .
\end{aligned}
$$

From a very early period in the history of the calculus of variations to the time of Weierstrass it was thought that an arc $C$ which made the first variation zero and the second variation positive for all choices of the function $\eta$, would surely minimize the integral $J$. The acceptance of this assumption, unjustified by any proof, was due to the analogy between the calculus of variations and the theory of maxima and minima of ordinary functions of ordinary variables. The assumption is correct in the case of a weak minimum, for which the only comparison curves admitted are those which are near to $C$ both in position and in direction, but Weierstrass showed that it is incorrect in the case of a strong minimum, for which the comparison curves are required to be close to $C$ in position only. He developed his necessary $E$-function condition, in addition to those which are deduced from the first and second variations, and proved that the conditions so found when slightly modified are sufficient to insure the existence of a minimum along the $\operatorname{arc} C$.

One result of this partial misconception by those who preceded Weierstrass was an elaborate theory of the second variation, the purpose of which was to reduce it to a form in which it would be clearly positive for all choices of the function

* An exposition is given by Bolza, Vorlesungen über Variationsrechnung, pp. $105 \mathrm{ff}$. 
$\eta(x)$. Legendre, in $1786,{ }^{*}$ was the first to attempt such a transformation. Euler, Lagrange, and other investigators before him had obtained from the first variation the differential equation of the minimizing curves, and conditions at the ends of the curves when the end points were allowed to vary. He himself begins his paper rather apologetically, it seems to me, his avowed purpose being to exhibit a criterion for distinguishing between extremal arcs which furnish maxima and those which furnish minima, whereas he says that in a majority of the applications one can usually readily decide this question from the setting of the problem itself. Inspired, however, by the analogous theory of maxima and minima of functions of a finite number of variables, he succeeded in transforming the second variation into the form

$$
J^{\prime \prime}(0)=\int_{x_{1}}^{x_{2}} f_{y^{\prime} y^{\prime}}\left(\eta^{\prime}+\frac{f_{y y^{\prime}}+\alpha}{f_{y^{\prime} y^{\prime}}} \eta\right)^{2} d x,
$$

where $\alpha$ is a solution of a certain Riccati differential equation of the first order. From this he concluded that the second variation will always be positive and therefore that the integral (1) will always be minimized by an extremal arc $C$ along which $f_{y^{\prime} y^{\prime}}>0$. His proof was faulty because there does not always exist a function $\alpha$ of the type he used, finite everywhere between the limits $x_{1}$ and $x_{2}$, but his conclusion that the positive form found for the second variation would ensure a minimum is correct for the case of a weak minimum, as I have already indicated. It turns out that the condition, $f_{y^{\prime} y^{\prime}} \geqq 0$ along the minimizing extremal arc, is necessary for a minimum but not always sufficient, and it is now called the Legendre necessary condition. It is interesting to note that Legendre himself was not entirely satisfied with his analysis, for he published later comments on his own paper in which he called attention to some of the weaknesses of his argument and suggested methods of overcoming them.

In 1837 Jacobi† announced the discovery of an important advance in the theory of the transformation of the second variation. He disclosed the circumstances under which the trans-

* "Abhandlung über die Untersuchung der Maxima und Minima in der Variations-Rechnung," Mémoires de l'Académie royale des Sciences, 1786, p. 7 .

$\dagger$ "Zur Theorie der Variationsrechnung und der Differentialgleichungen," Journal für die reine und angewandte Mathematik, vol. 17 (1837), p. 68 . 
formation of Legendre was possible and characterized the cases when it is not possible. On every extremal arc with a fixed initial point $A$ there is in general a point $A^{\prime}$ conjugate to $A$ such that Legendre's transformation is possible on every $\operatorname{arc} A B$ excluding $A^{\prime}$, but not possible on an arc $A B$ containing $A^{\prime}$. In justifying these statements Jacobi introduced, in place of the Riccati equation used by Legendre, a linear differential equation of the second order, now usually called the Jacobi differential equation, and he showed that its solutions can be obtained from those of Euler's equation by differentiations with respect to the constants of integration. He also used a new method for transforming the second variation which he applied successfully to the case when the integrand of the integral (1) contains higher derivatives of $y$. His conclusions were that an extremal arc $A B$ which contains the conjugate point $A^{\prime}$ either between $A$ and $B$ or at $B$ can not minimize the integral $J$, and that an extremal arc $A B$ which has $f_{y^{\prime} y^{\prime}}>0$ along it and which does not contain the conjugate point $A^{\prime}$ will surely minimize $J$.

Jacobi's results were correct with the exception of his statement that no minimum can exist when the point $A^{\prime}$ conjugate to $A$ coincides with $B$. It is now known that in this exceptional case there will sometimes be a minimum and sometimes not. But his proofs were again incomplete. I do not find in examining his paper that he justifies anywhere the necessity of the condition that the conjugate point $A^{\prime}$ shall not lie between $A$ and $B$, and his sufficiency proof rests solely upon his transformation of the second variation to positive form and is open to the same objection which I have mentioned before as applying to all of the writers who preceded Weierstrass.

In extending his transformation to integrals containing higher derivatives of the function $y(x)$ Jacobi made use of a theorem concerning linear differential equations which he did not prove. It is closely associated with the now well-known relation

$$
u J(v)-v J(u)=\frac{d}{d x} M(u, v)
$$

satisfied by a self-adjoint linear differential equation of the $n$th order. His incomplete exposition aroused much interest and led to a series of papers by Delaunay, Minding, Spitzer, 
Heine, Clebsch, Hesse, Lipschitz, Mayer, Scheffers, von Escherich, and others, who gave proofs of the Jacobi theorem and extended his results to more complicated problems of the calculus of variations. Of these papers I shall mention especially only those which seem to me to contain the more important advances.

In 1858 Clebsch* studied the transformation of the second variation for the very general type of problem formulated by Lagrange. For such a problem there is an integral of the form

$$
\int_{x_{1}}^{x_{2}} f\left(x, y_{1}, \cdots, y_{n}, y_{1}{ }^{\prime}, \cdots, y_{n}{ }^{\prime}\right) d x,
$$

to be minimized in the class of curves

$$
y_{i}=y_{i}(x) \quad\left(x_{1} \leqq x \leqq x_{2} ; i=1, \cdots, n\right)
$$

which join two fixed points and which satisfy besides a set of differential equations

$$
\varphi_{i}\left(x, y_{1}, \cdots, y_{n}, y_{1}{ }^{\prime}, \cdots, y_{n}{ }^{\prime}\right)=0 \quad(i=1, \cdots, m<n) .
$$

Clebsch found the system of linear differential equations of the second order analogous to the single Jacobi equation in the simpler case which we have been considering, and he expressed the second variation as an integral

$$
\int_{x_{1}}^{x_{2}} \sum_{i, k} F_{y_{i}^{\prime} y_{k}^{\prime}} W_{i} W_{k} d x
$$

In this expression $F$ is the sum

$$
F=f+\lambda_{1} \varphi_{1}+\cdots+\lambda_{m} \varphi_{m},
$$

the coefficients $\lambda_{i}$ being functions of $x$, and the variables $W_{i}$ are proportional to certain determinants of order $n+1$ which satisfy the relations

$$
\frac{\partial \varphi_{i}}{\partial y_{1}{ }^{\prime}} W_{1}+\cdots+\frac{\partial \varphi_{i}}{\partial y_{n}{ }^{\prime}} W_{n}=0 \quad(i=1, \cdots, m) .
$$

The Jacobi transformation of the second variation for the simpler problems is possible if there is a solution $u$ of

\footnotetext{
* Journal für die reine und angewandte Mathematik, vol. 55 (1858), pp. 254 and 335 ; in two papers: "Ueber die Reduktion der zweiten Variation auf ihre einfachste Form," "Ueber diejenigen Probleme der Variationsrechnung, welche nur eine unabhängige Variable enthalten."
} 
Jacobi's equation which does not vanish on the interval $x_{1} x_{2}$. For the Lagrange problem here under consideration there must be an $n$-square matrix with columns belonging to $n$ so-called conjugate systems of solutions of the Jacobi equations and having a determinant different from zero on $x_{1} x_{2}$.

The transformation of Clebsch seems to me a very complicated one. It is based upon a clever foresight of the form to which the integrand of the second variation may be reduced, and involves a count of the conditions necessary to make the reduction, the introduction of a sufficient number of arbitrary functions to satisfy these conditions, and the determination of the functions so introduced by a sequence of systems of equations. From the results of the transformation one may infer that the second variation will be positive whenever the quadratic form in the integrand of the expression (9) is positive at each point of the extremal arc under consideration. It is also provable that this property is necessary for a minimum, and the condition so found is sometimes called the LegendreClebsch condition because it is the generalization of the one which Legendre deduced for the simpler case.

Ten years after the papers of Clebsch appeared A. Mayer* presented the same results in somewhat simpler form and extended the Jacobi theory of the conjugate point to fit the Lagrange problem. He determined the position of the conjugate point by means of a zero of a determinant of solutions of Jacobi's equations, now called the Mayer determinant.

But the most thorough study of the theory of the transformation of the second variation is undoubtedly that of von Escherich. $\dagger$ In a series of five papers in 1898, 1899, and 1901 he effected the Clebsch transformation for several types of problems by a generalization of Jacobi's method, and studied in great detail the theory of conjugate points and the conjugate systems of solutions of Jacobi's equations which are important in the determination of these points as well as in the transformation itself. The equations which I. am designating here as the Jacobi equations, because they are generalizaitons of

\footnotetext{
* "Ueber die Kriterien des Maximums und Minimums der einfachen Integrale," Journal für die reine und angewandte Mathematik, vol. 69 (1868), p. 238 .

† "Die zweite Variation der einfachen Integrale," Sitzungsberichte der kaiserlichen Akademie der Wissenschaften in Wien, Mathematisch-naturwissenschaftliche Classe, vols. 107, 108, 110 (1898, 1899, 1901).
} 
the one which Jacobi used in the simpler case, are called by von Escherich the accessory system of linear differential equations. I will mention especially, without attempting to write it down, an important formula which lies at the basis of the von Escherich theory. It is a formula involving the determinants of conjugate systems and is of great assistance in the discussion of the zeros of the Mayer determinant which determines conjugate points. It should be added that von Escherich discovered, as a result of his detailed study, an important exceptional case for the Lagrange problem which he distinguishes from the more general normal or non-singular type. Most of his results apply only to the latter, and little is in fact known of the possibilities of the singular case.

A discussion of the second variation could not be complete without mentioning the work of Weierstrass. His results were obtained between 1865 and 1888 but they became known very slowly because he described them only in his lectures. The book by Kneser, Vorlesungen über Variationsrechnung, 1900, was I think the first published account of any importance. Weierstrass confined his attention for the most part to the problem in parametric form in the plane. His transformation of the second variation for this case was exceedingly ingenious, but apparently not readily generalizable to higher spaces. His other work, however, had also an important influence upon the study of the second variation. For the sufficiency proof which he developed for the case of a strong minimum applies also to a weak minimum, and a sufficiency proof in the latter case based on the theory of the transformation of the second variation is therefore superfluous.

Finally I should mention an interesting paper by Hahn* in which he shows the connection between the conjugate systems of solutions of Jacobi's equations and what are called Mayer fields. These latter are generalizations of the plane fields of which I have spoken above. For problems in higher spaces the analogue of the field covered by a one-parameter family of solutions of Euler's equations is an $n$-parameter family of extremals simply covering a region $F$ of space. But the Hilbert integral will be invariant in such a region $F$ only if the $n$-parameter family itself has special properties, in which case $F$ with its extremals is called a Mayer field

* "Ueber den Zusammenhang zwischen den Theorien der zweiten Variation und der Weierstrass'schen Theorie der Variationsrechnung," Rendiconti del Circolo Matematico di Palermo, vol. 29 (1910), p. 49. 
because Mayer was the first to recognize and study the properties of such families. The phenomena presented by the $n$-parameter families of Mayer fields are analogous to those of two-parameter families of straight lines in space. Not every such family is orthogonal to a surface. When one has this property it will form a Mayer field for the problem of minimizing the ordinary length integral in space. It turns out that every conjugate system of solutions of the Jacobi equations is deducible from the equations of the $n$-parameter family of a Mayer field by differentiation with respect to its parameters, and Hahn uses this property to assist in constructing the various types of conjugate systems which von Escherich studied.

I have now come to the results of which I wish especially to speak this afternoon. The historical sketch which I have given, though most incomplete, will suffice to show the great interest which mathematicians have taken in the theory of the second variation, and the complicated character of the transformations which have been devised for it. The results of Weierstrass mentioned above, and some very beautiful geometrical methods, developed by Darboux for geodesics and generalized by Kneser, enable one to deduce the necessary conditions of Legendre and Jacobi in some cases without the use of the complicated theory of the second variation. These proofs fail, however, when the enveloping curve upon which they depend has a singularity of a special type, and the efforts which have been made to complete the proofs to cover all cases have led to complications as great as those of the theory of the second variation itself.

The importance of the theory of the second variation at the present time lies therefore primarily in the proofs which it provides for the necessity of the analogues, for more general problems, of the conditions discovered by Legendre and Jacobi for the simpler cases. Furthermore the transformation of the second variation into a form clearly positive may be made the basis for the justification of a set of sufficient conditions for a weak minimum, and if the transformation can be effected simply the method will be an economical one. Finally I regard it as highly desirable from a historical point of view that the complicated theories which have been attached to the second variation should have an interpretation, in the light of recent results in the calculus of variations, which will make them more 
perspicuous and less detached than they seem to be at present from the theory as a whole.

For some years past I have been in the habit, in my lecture courses at the University of Chicago, of deducing the Legendre and Jacobi conditions for simpler problems by an application to the second variation of results described in the earlier part of this paper, the first two necessary conditions mentioned as deducible from the first variation and the Weierstrass $E$-function condition. The underlying thought is this. Since the second variation must always be positive or zero along a minimizing arc for all choices of the function $\eta(x)$ such that $\eta\left(x_{1}\right)=\eta\left(x_{2}\right)=0$, we are led to the consideration of a minimum problem in the $x \eta$-space for the integral (3) of the second variation. This problem is of precisely the same sort as the original problem of minimizing the integral (1). The curve $\eta=0$ is necessarily a minimizing curve for the $x \eta$-problem, when $J(C)$ is a minimum, since it gives the second variation its smallest value zero. Hence along it we must have the $E$-function condition satisfied,

$$
\begin{aligned}
\Omega\left(x, \eta, \bar{\eta}^{\prime}\right)-\Omega\left(x, \eta, \eta^{\prime}\right)-\left(\bar{\eta}^{\prime}-\eta^{\prime}\right) \Omega_{\eta^{\prime}}\left(x, \eta, \eta^{\prime}\right) & \\
& =f_{y^{\prime} y^{\prime}}\left(\bar{\eta}^{\prime}-\eta^{\prime}\right)^{2} \geqq 0,
\end{aligned}
$$

the first member of the equality being the Weierstrass $E$ function for the integrand function $\Omega$ of the second variation. It follows at once that the Legendre condition $f_{y^{\prime} y^{\prime}} \geqq 0$ must hold along the minimizing arc.

Furthermore, the Euler differential equation of this minimum problem of the second variation is the equation

$$
\Omega_{\eta}-\frac{d}{d x} \Omega_{\eta^{\prime}}=\left(f_{y y} \eta+f_{y y^{\prime} \eta^{\prime}}\right)-\frac{d}{d x}\left(f_{y^{\prime} y} \eta+f_{y^{\prime} y^{\prime} \eta^{\prime}}\right)=0
$$

which is precisely the linear differential equation of the second order in $\eta$ upon which Jacobi based his theory of the second variation. The coefficient of $\eta^{\prime \prime}$ in this equation is $f_{y^{\prime} y^{\prime}}$, and in discussing the Jacobi condition it is customary to presuppose this coefficient different from zero along the arc $C$, so that the solutions of the Jacobi equation will have no singularities on the interval $x_{1} x_{2}$. Suppose now that there is a solution $u$ of Jacobi's equation not identically zero but vanishing at $x_{1}$ and at a second point $x_{3}$ between $x_{1}$ and $x_{2}$. Then in the first place the value $u^{\prime}\left(x_{3}\right)$ is different from zero, since the 
only solution of Jacobi's equation vanishing with its derivative at any point whatsoever is $\eta \equiv 0$. Furthermore the function $\eta(x)$ defined by the conditions

$$
\begin{array}{lll}
\eta(x)=u(x) & \text { for } & x_{1} \leqq x \leqq x_{3} \\
\eta(x)=0 & \text { for } & x_{3} \leqq x \leqq x_{2}
\end{array}
$$

is an admissible function $\eta$. It is easily provable that $\eta(x)$ gives the second variation its minimum value zero, for with the help of an integration by parts and properties of the homogeneous quadratic function $\Omega$ we have

$$
\begin{aligned}
J^{\prime \prime}(0)=2 \int_{x_{1}}^{x_{3}} \Omega(x, & \left.u, u^{\prime}\right) d x=\int_{x_{1}}^{x_{3}}\left(u \Omega_{u}+u^{\prime} \Omega_{u^{\prime}}\right) d x \\
& =\left.u \Omega_{u^{\prime}}\right|_{x_{1}} ^{x_{3}}+\int_{x_{1}}^{x_{3}} u\left(\Omega_{u}-\frac{d}{d x} \Omega_{u^{\prime}}\right) d x=0 .
\end{aligned}
$$

The curve defined by $\eta(x)$ can not, however, be a minimizing curve for the second variation since it has a corner at $x_{3}$ at which

$$
\Omega_{\eta^{\prime}}\left(x_{3}-0\right)-\Omega_{\eta^{\prime}}\left(x_{3}+0\right)=\left.f_{y^{\prime} y^{\prime}} u^{\prime}\right|^{x_{3}} \neq 0 .
$$

Since $\eta(x)$ gives the second variation the value zero and does not minimize it, we conclude that there are variations $\bar{\eta}(x)$ giving the second variation a value less than zero, and it follows at once that the original integral $J$ can not be minimized by the arc $C$. We have then the result that if $J(C)$ is a minimum there is no solution $u$ 巫 of Jacobi's equation vanishing at $x_{1}$ and at a second point $x_{3}$ between $x_{1}$ and $x_{2}$. This is, however, exactly Jacobi's necessary condition, since the conjugate point to $A\left(x=x_{1}\right)$ is the point $A^{\prime}\left(x=x_{3}\right)$ corresponding to the first zero $x_{3}$ of $u(x)$ after $x_{1}$, and we see that arcs $A B$ containing $A^{\prime}$ between $A$ and $B$ have no longer the minimizing property.

It is to be noted that the proofs of the necessity of the Legendre and Jacobi conditions just described are made under the hypotheses usually presupposed when these conditions are deduced from the second variation, and I wish further to call attention to the fact that they do not involve any transformation of the second variation except the very simple one used in showing that the curve $\eta(x)$ makes the second variation vanish. The Euler differential equation condition, the corner 
point property, and the Weierstrass $E$-function condition, are properties of a minimizing arc which should be developed early in every complete discussion of the problem, and when we apply them to the second variation we are not using new methods peculiar to this later part of the theory. The effectiveness and economy of the methods just described are not as important for the simple case, which has been discussed in some detail, as they are for the more complicated problems of the calculus of variations. D. M. Smith* has, however, extended these methods so that they can be applied to the problem of Lagrange, and Miss G. A. Larew $\dagger$ has done the same for the problem of Mayer. I have myself modified them for problems in parametric form in higher spaces. $\$$ In these latter cases a transformation of the second variation analogous to that of Weierstrass for the plane seems now entirely unnecessary.

But it has seemed to me for some time that the theory of the second variation in its entirety could be viewed with success from the standpoint of the minimum problem of the second variation, a minimum problem within a minimum problem, so to speak, and I have recently satisfied myself that this is so for the Lagrange problem as well as for the simpler cases. A one-parameter family of extremals of the second variation simply covering a field in the $x \eta$-plane is a family of solutions of Jacobi's equations of the form

$$
\eta=a u \quad\left(x_{1} \leqq x \leqq x_{2}\right),
$$

where $u$ is a particular solution of the Jacobi equation which does not vanish on the interval $x_{1} x_{2}$, and $a$ is the parameter of the family. The slope function $\pi(x, \eta)$ for this field, expressing the slope of the extremal of the field through the point $(x, \eta)$ and corresponding to the slope function $p(x, y)$ of the general case, is found by solving the last equation for $a$ and substituting in the expression

$$
\pi(x, \eta)=a u^{\prime}=\eta \frac{u^{\prime}}{u} .
$$

* "Jacobi's condition for the problem of Lagrange in the calculus of variations," Trans. Amer. Math. Society, vol. 17 (1916), p. 459.

$\dagger$ "Necessary conditions in the problems of Mayer in the calculus of variations," Trans. Amer. Math. Society, vol. 20 (1919), p. 1.

‡"Jacobi's condition for problems of the calculus of variations in parametric form," Trans. Amer. Math. Society, vol. 17 (1916), p. 195. 
If $J^{\prime \prime}(H)$ represents the value of the second variation for an arbitrary function $\eta(x)$ vanishing at $x_{1}$ and $x_{2}$, and if $J^{\prime \prime}\left(H_{0}\right)$ is the corresponding value along $\eta=0$, then the formula (8) of Weierstrass when applied to the second variation takes the form

$$
\begin{aligned}
& J^{\prime \prime}(H)-J^{\prime \prime}\left(H_{0}\right) \\
& \quad=\int_{x_{1}}^{x_{2}}\left\{\Omega\left(x, \eta, \eta^{\prime}\right)-\Omega(x, \eta, \pi)-\left(\eta^{\prime}-\pi\right) \Omega_{\eta^{\prime}}(x, \eta, \pi)\right\} d x .
\end{aligned}
$$

But since $J^{\prime \prime}\left(H_{0}\right)=0$, an application of Taylor's formula to the integrand on the right gives

$$
J^{\prime \prime}(H)=\int_{x_{1}}^{x_{2}} f_{y^{\prime} y^{\prime}}\left(\eta^{\prime}-\pi\right)^{2} d x=\int_{x_{1}}^{x_{2}} f_{y^{\prime} y^{\prime}}\left(\frac{\eta^{\prime} u-\eta u^{\prime}}{u}\right)^{2} d x .
$$

This is precisely the form of the second variation found by Jacobi. From it we conclude at once that the second variation is positive or zero for every function $\eta$ vanishing at $x_{1}$ and $x_{2}$, provided that $f_{y^{\prime} y^{\prime}}>0$ along the original arc $C$, and that a solution $u$ of Jacobi's equation exists which is different from zero on the interval $x_{1} x_{2}$.

The analysis in the case of the Lagrange problem is of course more complicated. The one-parameter family (10) of the field for the second variation is here replaced by an $n$-parameter family

$$
\eta_{i}=a_{1} u_{i 1}+\cdots+a_{n} u_{i n} \quad(i=1, \cdots, n),
$$

for which the columns of the matrix ||$u_{i k}||$ belong to a conjugate system of solutions of the Jacobi equations. The slope functions $\pi_{i}(x, \eta)$ of the field are found by solving the last equations for the parameters $a_{i}$ and substituting them in the expressions

$$
\pi_{i}=a_{1} u_{i 1}{ }^{\prime}+\cdots+a_{n} u_{i n}{ }^{\prime} \quad(i=1, \cdots, n),
$$

where $u_{i k}{ }^{\prime}$ is the derivative of $u_{i k}$ with respect to $x$. The Weierstrass formula gives at once the value

$$
J^{\prime \prime}(H)=\int_{x_{1}}^{x_{2}} \sum_{i k} F_{y_{i}^{\prime} y_{k^{\prime}}}\left(\eta_{i}{ }^{\prime}-\pi_{i}\right)\left(\eta_{k}{ }^{\prime}-\pi_{k}\right) d x
$$

for the second variation, where $F$ has the significance before explained, and where the differences $\eta_{i}{ }^{\prime}-\pi_{i}$ turn out to 
have the values

$$
\eta_{i}{ }^{\prime}-\pi_{i}=W_{i}=\frac{1}{\left|u_{i k}\right|} \chi_{i}(\eta)
$$

in terms of the functions $W_{i}$ of Clebsch and the determinants $\chi_{i}(\eta)$ of von Escherich. These latter functions have hitherto appeared in somewhat artificial fashion in the theory and it seems to me interesting to have this very simple interpretation of them in terms of the slope functions of the field.

The von Escherich fundamental formula which I mentioned above* may be expressed by the equation

$$
\sum_{i \bar{k}} F_{y_{i}{ }^{\prime} y^{\prime}}\left(\eta_{i}{ }^{\prime}-\pi_{i}\right)\left(\eta_{k}{ }^{\prime}-\pi_{k}\right)=\sum_{i} a_{i}{ }^{\prime} \psi_{i},
$$

where the symbols $a_{i}{ }^{\prime}$ represent the derivatives of the solutions $a_{i}$ of the equations (11) with respect to $x$ when the variables $\eta_{i}$ are thought of as functions of $x$, and the coefficients $\psi_{i}$ are bilinear expressions in the elements of two sets of functions $\left(\eta_{1}, \cdots, \eta_{n} ; \mu_{1}, \cdots, \mu_{m}\right)$ and $\left(u_{1 i}, \cdots, u_{n i} ; \sigma_{1 i}, \cdots, \sigma_{m i}\right)$, the latter of which is the system of solutions of Jacobi's equations to which the column $u_{1 i}, \cdots, u_{n i}$ of the matrix ||$u_{i k}||$ belongs. If the set $(\eta ; \mu)$ is also a solution of Jacobi's equations then each of these coefficients is a constant. For this special case I have already published a proof $\dagger$ of the von Escherich formula, but the one which I have mentioned here is quite general and simpler than my earlier one.

Finally the von Escherich theory of conjugate systems of solutions of Jacobi's equations is identical with the theory of Mayer fields for the minimum problem of the second variation, and the methods which Hahn devised for relating conjugate systems to the Mayer fields of the original minimum problem can be used to construct the various types of conjugate systems which von Escherich considered.

In conclusion I may say that the methods suggested above seem to me of considerable assistance in developing and understanding the theory of the calculus of variations. For in the first place they enable one to retain the advantages of the second variation, in deducing the necessary conditions analogous to those of Legendre and Jacobi, without becoming involved in elaborate transformations. In the second place

* Von Escherich, loc. cit., Mittheilung IV, Section XXIII, formula (9).

+ "A note on the problem of Lagrange in the calculus of variations," this Bulletin, vol. 22 (1916), p. 220. 
they may give some satisfaction to those who have delved in the theory of those transformations, since they make the transformations appear, not as separate chapters of the analysis, but as special applications of formulas now well established in other parts of the theory. It is even possible to make a satisfactory and not over complicated sufficiency proof for a weak minimum, without the use of the Weierstrass notion of a field. For the Weierstrass formula can be proved directly with some ease for the second variation when once it has been seen to hold true for a conjugate system of solutions of Jacobi's equations. It is not strange that the second variation has not been attacked from this standpoint before in spite of the fact that in my recent review of the literature I have found several suggestions which might have instigated one to attempt it. The real reason is, I think, that the advances of Weierstrass and Hilbert were published after 1900 and about the time that Kneser found his envelope theorem. The tendency since then has been to discard the theory of the second variation in favor of the more geometrical theory, but the experiment, so far as I know, has not been completely successful.

The University of Chicago, December, 1919.

\title{
GROUPS GENERATED BY TWO OPERATORS OF ORDER THREE WHOSE PRODUCT IS OF ORDER FOUR.
}

\author{
BY PROFESSOR G. A. MILLER.
}

(Read before the American Mathematical Society December 30,1919.)

\section{§1. Introduction.}

IT is known that the only groups which are completely determined by the orders of two generators and the order of their product are the dihedral groups and the groups of movements of the five regular solids known to the ancients. In all other cases two generators which are not restricted except as regards their orders and the order of their product 